\title{
COMMON EXPANSIONS IN NONINTEGER BASES
}

\author{
VILMOS KOMORNIK AND ATTILA PETHỐ
}

Dedicated to Professor Zoltán Daróczy on his 75th birthday.

\begin{abstract}
In this paper we study the existence of simultaneous representations of real numbers in bases $p>q>1$ with the digit set $A=\{-m, \ldots, 0, \ldots, m\}$. Among other results, we prove if $m=1$ and $q<2$, then there is a continuum of sequences $\left(c_{i}\right) \in A^{\infty}$ satisfying $\sum_{i=1}^{\infty} c_{i} q^{-i}=\sum_{i=1}^{\infty} c_{i} p^{-i}$. On the other hand, if $m=1$ and $q \geq 2+\sqrt{2}$, then only the trivial sequence $\left(c_{i}\right)=0^{\infty}$ satisfies the former equality.
\end{abstract}

\section{INTRODUCTION}

Given a finite alphabet or digit set $A$ of real numbers and a real base $q>1$, by an expansion of a real number $x$ we mean a sequence $c=\left(c_{i}\right) \in A^{\infty}$ satisfying the equality

$$
\sum_{i=1}^{\infty} \frac{c_{i}}{q^{i}}=x .
$$

This concept was introduced by Rényi [10] as a generalization of the radix representation of integers.

Given two different bases $p, q$ we wonder whether there exist real numbers having the same expansions in both bases:

$$
\sum_{i=1}^{\infty} \frac{c_{i}}{q^{i}}=x=\sum_{i=1}^{\infty} \frac{c_{i}}{p^{i}} .
$$

In case $0 \in A$ a trivial example is $x=0$ with $\left(c_{i}\right)=0^{\infty}$. If the alphabet $A$ contains no pair of digits with opposite signs, then this is the only such example. Indeed, if for instance all digits are nonnegative and

Date: Version of 2014-05-08-a.

1991 Mathematics Subject Classification. 11A63, 11B83.

Key words and phrases. simultaneous Rényi expansion, interval filling sequences.

The second author was partially supported by the OTKA grants No. 100339 and 104208 . 
$0^{\infty} \neq\left(c_{i}\right) \in A^{\infty}$, then for $p>q$ we have

$$
\sum_{i=1}^{\infty} \frac{c_{i}}{p^{i}}<\sum_{i=1}^{\infty} \frac{c_{i}}{q^{i}}
$$

by an elementary monotonicity argument.

Even if the alphabet $A$ contains digits of opposite signs, the existence of common expansions (1) seems to be a rare event.

Similar phenomena appears with the common radix representation. Indlekofer, Kátai and Racskó [4] called $\mathbf{a} \in \mathbb{Z}^{d}$ simultaneously representable by $\mathbf{q} \in \mathbb{Z}^{d}$, if there exist integers $0 \leq m_{0}, \ldots, m_{\ell}<Q:=$ $\left|q_{1} \cdots q_{d}\right|$ such that

$$
a_{i}=\sum_{j=0}^{\ell} m_{j} q_{i}^{j}, \quad i=1, \ldots, d .
$$

If $q_{1}, \ldots, q_{d}>0$ then apart from the zero vector no integer vectors are simultaneously representable by q. If, however, some of the base numbers are negative, then simultaneous representations may appear. For example take $q_{1}=-2$ and $q_{2}=-3$ then we have $(101)_{10}=$ $(1431335045)_{-2}=(1431335045)_{-3}$. Changing the sign of the "digits" with odd position we get a common representation of 101 in bases 2 and 3 with digits from $\{-6, \ldots, 0, \ldots, 6\}$. Pethő [8] gave a criterion of simultaneous representability on the one hand with the Chinese reminder theorem and, on the other hand with CNS polynomials. A similar result was proved by Kane [7].

No results on simultaneous representability of real numbers in noninteger bases seem to have appeared in the literature. In this paper we start such a study by investigating the case of the special alphabets $A=\{-m, \ldots, 0, \ldots, m\}$ for some given integer $m \geq 1$. Let us denote by $C(p, q)$ the set of sequences $\left(c_{i}\right) \in A^{\infty}$ satisfying

$$
\sum_{i=1}^{\infty} \frac{c_{i}}{q^{i}}=\sum_{i=1}^{\infty} \frac{c_{i}}{p^{i}} .
$$

We call $C(p, q)$ trivial if its only element is the null sequence.

Our main result is the following:

Theorem 1. Let $p>q>1$.

(i) If $q<(1+\sqrt{8 m+1}) / 2$, then $C(p, q)$ has the cardinality of the continuum.

(ii) If $(1+\sqrt{8 m+1}) / 2 \leq q \leq m+1$, then $C(p, q)$ is infinite.

(iii) Let $m+1<q \leq 2 m+1$. 
(a) If

$$
p \leq \frac{(m+1)(q-1)}{q-m-1},
$$

then $C(p, q)$ is nontrivial.

(b) If

$$
p>\frac{(m+1)(q-1)}{q-m-1},
$$

then $C(p, q)$ is trivial.

(iv) Let $2 m+1<q<m+1+\sqrt{m(m+1)}$.

(a) $C(p, q)$ is a finite set.

(b) There is a continuum of values $p>q$ for which $C(p, q)$ is nontrivial.

(c) If $p>q$ satisfies (4), then $C(p, q)$ is trivial.

(v) If $q \geq m+1+\sqrt{m(m+1)}$, then $C(p, q)$ is trivial.

Remark 2.

(i) The proof of (iii) (a) will also show that if $m+1<q \leq 2 m+1$, and

$$
\frac{1}{m} \leq \frac{1}{q-1}+\left(\frac{1}{q-1}-\frac{1}{p-1}\right) \frac{q^{n}}{p^{n}-q^{n}}
$$

for some positive integer $n$, then $C(p, q)$ has at least $n+1$ elements. For $n=1$ this condition reduces to (3).

Furthermore, we show in Remark 7 that the right side of this inequality is a decreasing function of $p$, so that the solutions $p$ of the inequality form a half-closed interval, say $\left(q, p_{n}\right]$. (We have clearly $p_{1}>p_{2}>\cdots$.)

(ii) The proof of (iv) (a) will show more precisely that if $q>2 m+1$ and

$$
\frac{1}{2 m}>\frac{1}{q-1}+\left(\frac{1}{q-1}-\frac{1}{p-1}\right) \frac{q^{n}}{p^{n}-q^{n}}
$$

for some positive integer $n$, then $C(p, q)$ has at most $(2 m+1)^{n}$ elements.

\section{PROOFS}

We begin by establishing some auxiliary results.

Interval filling sequences play an important role in establishing the existence of various kinds of representations of real numbers; see, e.g., Daróczy, Járai and Kátai [1], Daróczy and Kátai [2]. We also need 
such a result here: a variant of a classical theorem of Kakeya [5], [6] (see also [9, Part 1, Exercise 131]).

Proposition 3. Let $A=\{-m, \ldots, 0, \ldots, m\}$ and let $\sum_{k=1}^{\infty} r_{k}$ be a convergent series of positive numbers, satisfying the inequalities

$$
r_{n} \leq 2 m \sum_{k=n+1}^{\infty} r_{k}
$$

for all $n=1,2, \ldots$ Then the sums

$$
\sum_{k=1}^{\infty} c_{k} r_{k}, \quad\left(c_{k}\right) \in A^{\infty}
$$

fill the interval

$$
\left[-m \sum_{k=1}^{\infty} r_{k}, m \sum_{k=1}^{\infty} r_{k}\right] .
$$

Proof. It is clear that all sums (7) belong to the interval (8). Conversely, for each given $x$ in this interval we define a sequence $\left(c_{k}\right) \in A^{\infty}$ by the following greedy algorithm. If $c_{1}, \ldots, c_{n-1}$ are already defined (no assumption if $n=1$ ), then let $c_{n}$ be the largest element of $A$ such that

$$
\left(\sum_{k=1}^{n} c_{k} r_{k}\right)-m\left(\sum_{k=n+1}^{\infty} r_{k}\right) \leq x .
$$

Letting $n \rightarrow \infty$ it follows that $\sum_{k=1}^{\infty} c_{k} r_{k} \leq x$. It remains to prove the converse inequality. This is obvious if $c_{k}=m$ for all $k \in \mathbb{N}$ because then

by the choice of $x$.

$$
\sum_{k=1}^{\infty} c_{k} r_{k}=m \sum_{k=1}^{\infty} r_{k} \geq x
$$

If $c_{n}<m$ for infinitely many indices, then

$$
\left(\sum_{k=1}^{n-1} c_{k} r_{k}\right)+m r_{n}-m\left(\sum_{k=n+1}^{\infty} r_{k}\right)>x
$$

for all such indices, and letting $n \rightarrow \infty$ we conclude that $\sum_{k=1}^{\infty} c_{k} r_{k} \geq x$.

The proof will be complete if we show that $\left(c_{k}\right)$ cannot have a last term $c_{n}<m$, i.e., an index $n$ such that $c_{n}=j<m$, and $c_{k}=m$ for all $k>n$. Assume on the contrary that there exists such an index $n$. Then we have

$$
\left(\sum_{k=1}^{n-1} c_{k} r_{k}\right)+j r_{n}+m\left(\sum_{k=n+1}^{\infty} r_{k}\right) \leq x
$$


and

$$
\left(\sum_{k=1}^{n-1} c_{k} r_{k}\right)+(j+1) r_{n}-m\left(\sum_{k=n+1}^{\infty} r_{k}\right)>x
$$

by construction. Hence

$$
r_{n}>2 m \sum_{k=n+1}^{\infty} r_{k}
$$

contradicting (6).

We also need two technical lemmas.

Lemma 4. If $1<q<(1+\sqrt{8 m+1}) / 2$ and $p>q$, then the sequence $\left(r_{k}\right)_{k \in \mathbb{N}}:=\left(q^{-i}-p^{-i}\right)_{i \in \mathbb{N} \backslash n \mathbb{N}}$ satisfies (6) for all sufficiently large integers $n$.

Proof. Fix a sufficiently large integer $n$ such that

$$
\frac{1}{2 m}<\frac{1}{q(q-1)}-\frac{1}{q\left(q^{n}-1\right)}
$$

This is possible by our assumption on $q$, because we have the following equivalences for $m>0$ and $q>1$ :

$$
\begin{aligned}
\frac{1}{2 m}<\frac{1}{q(q-1)} & \Longleftrightarrow 4 q(q-1)<8 m \\
& \Longleftrightarrow(2 q-1)^{2}<8 m+1 \\
& \Longleftrightarrow 2 q-1<\sqrt{8 m+1} \\
& \Longleftrightarrow q<(1+\sqrt{8 m+1}) / 2 .
\end{aligned}
$$

Now, if

$$
r_{h^{\prime}}=q^{-h}-p^{-h}=q^{-h}\left(1-(q / p)^{h}\right)
$$

for some $h^{\prime} \geq 1$, then

$$
\sum_{k=h^{\prime}+1}^{\infty} r_{k}=\sum_{i \in \mathbb{N} \backslash n \mathbb{N}, i>h}\left(q^{-i}-p^{-i}\right)=\sum_{i \in \mathbb{N} \backslash n \mathbb{N}, i>h} q^{-i}\left(1-(q / p)^{i}\right) .
$$


Since $\left(1-(q / p)^{i}\right)>\left(1-(q / p)^{h}\right)$ for all $i>h$, it follows that (we use the choice of $n$ in the last step)

$$
\begin{aligned}
\frac{\sum_{k=h^{\prime}+1}^{\infty} r_{k}}{r_{h^{\prime}}} & \geq \sum_{i \in \mathbb{N} \backslash n \mathbb{N}, i>h} q^{h-i} \\
& =\left(\sum_{i=1}^{\infty} q^{-i}\right)-\left(\sum_{i>\frac{h}{n}} q^{h-i n}\right) \\
& \geq\left(\sum_{i=1}^{\infty} q^{-i}\right)-\left(\sum_{i=0}^{\infty} q^{-1-i n}\right) \\
& =\left(\sum_{i=2}^{\infty} q^{-i}\right)-\left(\sum_{i=1}^{\infty} q^{-1-i n}\right) \\
& =\frac{1}{q(q-1)}-\frac{1}{q\left(q^{n}-1\right)}>\frac{1}{2 m} .
\end{aligned}
$$

Lemma 5. Let $p>q>1$. The sequence

$$
\left(\frac{\sum_{i=n+1}^{\infty}\left(q^{-i}-p^{-i}\right)}{q^{-n}-p^{-n}}\right)_{n=1}^{\infty}
$$

is strictly decreasing, and tends to $1 /(q-1)$.

Proof. Since $1>(q / p)^{n} \searrow 0$, the results follow from the identity

$$
\frac{\sum_{i=n+1}^{\infty}\left(q^{-i}-p^{-i}\right)}{q^{-n}-p^{-n}}=\frac{1}{q-1}+\frac{p-q}{(q-1)(p-1)} \frac{(q / p)^{n}}{1-(q / p)^{n}}
$$

Setting $x=q / p$ for brevity, the identity is proved as follows:

$$
\begin{aligned}
\frac{\sum_{i=n+1}^{\infty}\left(q^{-i}-p^{-i}\right)}{q^{-n}-p^{-n}} & =\frac{q^{-n}}{(q-1)\left(q^{-n}-p^{-n}\right)}-\frac{p^{-n}}{(p-1)\left(q^{-n}-p^{-n}\right)} \\
& =\frac{1}{(q-1)\left(1-x^{n}\right)}-\frac{x^{n}}{(p-1)\left(1-x^{n}\right)} \\
& =\frac{1-x^{n}+x^{n}}{(q-1)\left(1-x^{n}\right)}-\frac{x^{n}}{(p-1)\left(1-x^{n}\right)} \\
& =\frac{1}{q-1}+\frac{x^{n}}{1-x^{n}}\left(\frac{1}{q-1}-\frac{1}{p-1}\right) \\
& =\frac{1}{q-1}+\frac{p-q}{(q-1)(p-1)} \frac{x^{n}}{1-x^{n}} .
\end{aligned}
$$


Remark 6. Let us note for further reference the following equivalent form of (9), obtained during the proof:

$$
\frac{\sum_{i=n+1}^{\infty}\left(q^{-i}-p^{-i}\right)}{q^{-n}-p^{-n}}=\frac{1}{q-1}+\left(\frac{1}{q-1}-\frac{1}{p-1}\right) \frac{q^{n}}{p^{n}-q^{n}} .
$$

Now we are ready to prove our theorem.

Proof of Theorem 1 (i). We adapt the proof of Theorem 3 in [3], which states that if $1<q<(1+\sqrt{5}) / 2$, then every $x$ satisfying $q<x<$ $1 /(q-1)$ has a continuum of expansions in base $q$ with digits 0 or 1 .

Applying Lemma 4 we fix a large positive integer $n$ such that the sequence $\left(r_{k}\right)_{k \in \mathbb{N}}:=\left(q^{-i}-p^{-i}\right)_{i \in \mathbb{N} \backslash n \mathbb{N}}$ satisfies (6). Next we fix a large positive integer $N$ such that

$$
\begin{gathered}
{\left[-m \sum_{i=N}^{\infty}\left(q^{-i n}-p^{-i n}\right), m \sum_{i=N}^{\infty}\left(q^{-i n}-p^{-i n}\right)\right]} \\
\subset\left[-m \sum_{i \in \mathbb{N} \backslash n \mathbb{N}}\left(q^{-i n}-p^{-i n}\right), m \sum_{i \in \mathbb{N} \backslash n \mathbb{N}}\left(q^{-i n}-p^{-i n}\right)\right] .
\end{gathered}
$$

This is possible because the right side interval contains 0 in its interior. The sets

$$
\begin{aligned}
& B:=\mathbb{N} \backslash n \mathbb{N}, \\
& C:=\{i n: i=N, N+1, \ldots\}, \\
& D:=\{i n: i=1, \ldots, N-1\}
\end{aligned}
$$

form a partition of $\mathbb{N}$.

Choose an arbitrary sequence $\left(c_{i}\right)_{i \in C} \in A^{C}$; there is a continuum of such sequences because $C$ is an infinite set. Since

$$
-\sum_{i \in C} c_{i}\left(q^{-i}-p^{-i}\right)
$$

belongs to the left side interval in (11), applying Proposition 3 there exists a sequence $\left(c_{i}\right)_{i \in B} \in A^{B}$ such that

$$
\sum_{i \in B \cup C} c_{i}\left(q^{-i}-p^{-i}\right)=0 .
$$

Setting $c_{i}=0$ for $i \in D$ we obtain a sequence $\left(c_{i}\right)_{i \in \mathbb{N}} \in C(p, q)$.

Proof of Theorem 1 (ii). We show that for each positive integer $n$ there exists a sequence $\left(c_{i}\right) \in C(p, q)$, beginning with $c_{1}=\cdots=c_{n-1}=0$ 
and $c_{n}=-1$. Indeed, since $q \leq m+1$, by Lemma 5 we have

$$
0<q^{-n}-p^{-n}<(q-1) \sum_{i=n+1}^{\infty}\left(q^{-i}-p^{-i}\right) \leq m \sum_{i=n+1}^{\infty}\left(q^{-i}-p^{-i}\right) .
$$

Since $q \leq 2 m+1$, Lemma 5 also shows that the condition (6) of Proposition 3 is satisfied for the alphabet $A=\{-m, \ldots, m\}$ and the sequence $r_{k}:=q^{-k-n}-p^{-k-n}, k=1,2, \ldots$ Hence there exists a sequence $\left(c_{i}\right)_{i=n+1}^{\infty} \in A^{\infty}$ satisfying

$$
q^{-n}-p^{-n}=\sum_{i=n+1}^{\infty} c_{i}\left(q^{-i}-p^{-i}\right)
$$

setting $c_{1}=\cdots=c_{n-1}=0$ and $c_{n}=-1$ this yields (2).

Proof of Theorem 1 (iii) (a). We show that there is a sequence $\left(c_{i}\right) \in$ $C(p, q)$, beginning with $c_{1}=-1$. Since $q \leq 2 m+1$, by Proposition 3 and Lemma 5 it is sufficient to show that

$$
(0<) q^{-1}-p^{-1} \leq m \sum_{i=2}^{\infty}\left(q^{-i}-p^{-i}\right) .
$$

By (9) this is equivalent to the inequality

$$
\frac{1}{m} \leq \frac{1}{q-1}+\frac{p-q}{(p-1)(q-1)} \frac{\frac{q}{p}}{1-\frac{q}{p}}=\frac{1}{q-1}+\frac{q}{(p-1)(q-1)}
$$

i.e., to $p \leq(m+1)(q-1) /(q-m-1)$. Indeed, since $m>0, q>1$ and $p>m+1$, we have

$$
\begin{aligned}
\frac{1}{m} \leq \frac{1}{q-1}+\frac{q}{(p-1)(q-1)} & \Longleftrightarrow(p-1)(q-1) \leq m(p-1)+m q \\
& \Longleftrightarrow p(q-m-1) \leq(m+1)(q-1) \\
& \Longleftrightarrow p \leq \frac{(m+1)(q-1)}{q-m-1} .
\end{aligned}
$$

Remark 7. Now we prove our statement in Remark 2 (i). If $m+1<$ $q \leq 2 m+1$ and $p>q$ is closer to $q$ so that

$$
(0<) q^{-n}-p^{-n} \leq m \sum_{i=n+1}^{\infty}\left(q^{-i}-p^{-i}\right)
$$

or equivalently (see (10))

$$
\frac{1}{m} \leq \frac{1}{q-1}+\left(\frac{1}{q-1}-\frac{1}{p-1}\right) \frac{q^{n}}{p^{n}-q^{n}}
$$


for some positive integer $n$, then the adaptation of the preceding proof shows that for each $k=1, \ldots, n$ there exists a sequence $\left(c_{i}\right) \in C(p, q)$, beginning with $c_{1}=\cdots=c_{k-1}=0$ and $c_{k}=-1$.

The right side of the above inequality is a decreasing function of $p$ because the function

$$
f(p):=\left(\frac{1}{q-1}-\frac{1}{p-1}\right) \frac{1}{p^{n}-q^{n}}
$$

has a negative derivative for all $p>q$.

Indeed, we have

$$
f^{\prime}(p)=\frac{1}{(p-1)^{2}\left(p^{n}-q^{n}\right)}-\left(\frac{1}{q-1}-\frac{1}{p-1}\right) \frac{n p^{n-1}}{\left(p^{n}-q^{n}\right)^{2}},
$$

whence

$$
\frac{(p-1)^{2}\left(p^{n}-q^{n}\right)^{2}}{p-q} f^{\prime}(p)=\frac{p^{n}-q^{n}}{p-q}-n p^{n-1} \frac{p-1}{q-1}<\frac{p^{n}-q^{n}}{p-q}-n p^{n-1} .
$$

We conclude by noticing that $\frac{p^{n}-q^{n}}{p-q}=n r^{n-1}$ by the Lagrange mean value theorem for some $q<r<p$ and therefore

$$
\frac{p^{n}-q^{n}}{p-q}-n p^{n-1}=n\left(r^{n-1}-p^{n-1}\right) \leq 0 .
$$

Proof of Theorem 1 (iv) (a). Since $1 /(q-1)<1 / 2 m$, by Lemma 5 we have

$$
q^{-n}-p^{-n}>2 m \sum_{i=n+1}^{\infty}\left(q^{-i}-p^{-i}\right)
$$

for all sufficiently large integers $n$, say for all $n>N{ }^{1}$ This implies that if two different sequences $\left(c_{i}\right),\left(c_{i}^{\prime}\right) \in A^{\infty}$ satisfy $c_{i}=c_{i}^{\prime}$ for $i=1, \ldots, N$, then

$$
\sum_{i=1}^{\infty} c_{i}\left(q^{-i}-p^{-i}\right) \neq \sum_{i=1}^{\infty} c_{i}^{\prime}\left(q^{-i}-p^{-i}\right)
$$

\footnotetext{
${ }^{1}$ If this inequality holds for some $n$, then it also holds for all larger integers by the monotonicity property of Lemma 5 .
} 
Indeed, if $n$ is the first index for which $c_{n} \neq c_{n}^{\prime}$, then $n>N$, and therefore

$$
\begin{aligned}
& \left|\sum_{i=1}^{\infty}\left(c_{i}-c_{i}^{\prime}\right)\left(q^{-i}-p^{-i}\right)\right| \\
& \quad \geq\left|c_{n}-c_{n}^{\prime}\right|\left(q^{-n}-p^{-n}\right)-\sum_{i=n+1}^{\infty}\left|c_{i}-c_{i}^{\prime}\right|\left(q^{-i}-p^{-i}\right) \\
& \quad \geq\left(q^{-n}-p^{-n}\right)-2 m \sum_{i=n+1}^{\infty}\left(q^{-i}-p^{-i}\right) \\
& \quad>0 .
\end{aligned}
$$

It follows that if two different sequences $\left(c_{i}\right),\left(c_{i}^{\prime}\right) \in A^{\infty}$ satisfy

$$
\sum_{i=1}^{\infty} \frac{c_{i}}{q^{i}}-\sum_{i=1}^{\infty} \frac{c_{i}}{p^{i}}=\sum_{i=1}^{\infty} \frac{c_{i}^{\prime}}{q^{i}}-\sum_{i=1}^{\infty} \frac{c_{i}^{\prime}}{p^{i}}=0
$$

then already their beginning words $c_{1} \ldots c_{N}$ and $c_{1}^{\prime} \ldots c_{N}^{\prime}$ must differ. We conclude that there are at most $(2 m+1)^{N}$ sequences $\left(c_{i}\right) \in A^{\infty}$ satisfying (2).

Proof of Theorem 1 (iv) (b). Thanks to (a) it is sufficient to exhibit a continuum of sequences $\left(c_{i}\right) \in A^{\infty}$ such that each sequence satisfies (2) for at least one base $p>q$.

Our assumption $q<m+1+\sqrt{m(m+1)}$ implies the inequality

$$
\frac{1}{q^{2}}<m \sum_{i=2}^{\infty} \frac{i}{q^{i+1}} .
$$

Indeed, differentiating the identity

$$
\sum_{i=1}^{\infty} \frac{1}{q^{i}}=\frac{1}{q-1}
$$

we get

$$
\sum_{i=1}^{\infty} \frac{i}{q^{i+1}}=\frac{1}{(q-1)^{2}}
$$

so that, since $m>0$ and $q>1$, (12) is equivalent to

$$
\frac{m+1}{q^{2}}<\frac{m}{(q-1)^{2}} .
$$

This inequality can be rewritten as

$$
q^{2}-2 q(m+1)+m+1<0 .
$$


The polynomial $x^{2}-2 x(m+1)+m+1$ has exactly one root, which is larger than one, namely $x=m+1+\sqrt{m(m+1)}$. Thus (12) holds if and only if $q<m+1+\sqrt{m(m+1)}$.

In view of (12) we may choose a sufficiently large positive integer $N$ such that

$$
\frac{1}{q^{2}}<m \sum_{i=2}^{N} \frac{i}{q^{i+1}}
$$

Now fix an arbitrary sequence $\left(c_{i}\right) \in A^{\infty}$ satisfying

$$
c_{1}=-1, c_{2}=\cdots=c_{N}=m \text { and } c_{i} \geq 0 \text { for all } i>N .
$$

(There is a continuum of such sequences.) We are going to prove that

(2) holds for at least one base $p>q$.

It is sufficient to show that

$$
\sum_{i=1}^{\infty} c_{i}\left(q^{-i}-p^{-i}\right)<0
$$

if $p>q$ is large enough, and

$$
\sum_{i=1}^{\infty} c_{i}\left(q^{-i}-p^{-i}\right)>0
$$

if $p>q$ is close enough to $q$. Indeed, then we will have equality for some intermediate value of $p$ by continuity.

The first property will follow from the stronger relation

$$
\lim _{p \rightarrow \infty} \sum_{i=1}^{\infty} c_{i}\left(q^{-i}-p^{-i}\right)<0, \quad \text { i.e., } \quad \sum_{i=1}^{\infty} \frac{c_{i}}{q^{i}}<0 .
$$

The proof is straightforward: since $c_{1}=-1$ and $q>m+1$, we have

$$
\sum_{i=1}^{\infty} \frac{c_{i}}{q^{i}} \leq \frac{-1}{q}+\sum_{i=2}^{\infty} \frac{m}{q^{i}}=\frac{-1}{q}+\frac{m}{q(q-1)}<\frac{-1}{q}+\frac{1}{q}=0 .
$$

Since $c_{i} \geq 0$ for all $i>N$, the second property is weaker than the inequality

$$
\sum_{i=1}^{N} c_{i}\left(q^{-i}-p^{-i}\right)>0
$$

for all $p>q$ close enough to $q$, and this is weaker than the relation

$$
\lim _{p \rightarrow q} \frac{1}{p-q} \sum_{i=1}^{N} c_{i}\left(q^{-i}-p^{-i}\right)>0 \text {. }
$$


The last property follows by using (14) and (15):

$$
\lim _{p \rightarrow q} \frac{1}{p-q} \sum_{i=1}^{N} c_{i}\left(q^{-i}-p^{-i}\right)=\sum_{i=1}^{N} \frac{i c_{i}}{q^{i+1}}=-\frac{1}{q^{2}}+m \sum_{i=2}^{N} \frac{i}{q^{i+1}}>0 .
$$

Proof of Theorem 1 (iii) (b), (iv) (c) and (v). If $p>q>m+1$ satisfy (4), then the proof of (iii) (a) shows that

$$
q^{-1}-p^{-1}>m \sum_{i=2}^{\infty}\left(q^{-i}-p^{-i}\right)
$$

Then by Lemma 5 we also have, more generally,

$$
q^{-n}-p^{-n}>m \sum_{i=n+1}^{\infty}\left(q^{-i}-p^{-i}\right)
$$

for all positive integers $n$.

Now if a sequence $\left(c_{i}\right) \in A^{\infty}$ has a first nonzero term $c_{n}$, then

$$
\begin{aligned}
&\left|\sum_{i=n+1}^{\infty} c_{i}\left(q^{-i}-p^{-i}\right)\right| \leq \sum_{i=n+1}^{\infty} m\left(q^{-i}-p^{-i}\right) \\
&<q^{-n}-p^{-n} \leq\left|c_{n}\left(q^{-n}-p^{-n}\right)\right|,
\end{aligned}
$$

so that (2) cannot hold. This completes the proof of (iii) (b) and (iv) (c).

For the proof of $(\mathrm{v})$ it remains to check that in case $q \geq m+1+$ $\sqrt{m(m+1)}$ the condition (4) holds for all $p>q$. This is equivalent to

$$
q \geq \frac{(m+1)(q-1)}{q-m-1},
$$

which can be rewritten as

$$
q^{2}-2 q(m+1)+m+1 \geq 0 .
$$

By our observation after (13) this inequality holds if and only if $q \geq$ $m+1+\sqrt{m(m+1)}$.

We end this paper by formulating some open questions:

(1) Find the optimal conditions on $p$ and $q$ in Theorem 1. In particular,

(a) Can $C(p, q)$ be infinite for some $p>q>m+1$ ?

(b) In case $2 m+1<q<m+1+\sqrt{m(m+1)}$ is $C(p, q)$ nontrivial for all $p>q$ sufficiently close to $q$ ? 
(2) Construct an alphabet and three (or more) different bases such that a continuum of (or infinitely many) real numbers have identical expansions in all three bases.

(3) Given two bases $p>q>1$ investigate the set of points of the form

$$
\sum_{i=1}^{\infty} c_{i}\left(p^{-i}-q^{-i}\right), \quad\left(c_{i}\right) \in A^{\infty} .
$$

Acknowledgement. The authors thank the referees for their suggestions to improve the presentation of the paper.

\section{REFERENCES}

[1] Z. Daróczy, A. Járai and I. Kátai, Intervallfüllende Folgen und volladditive Funktionen, Acta Sci. Math. (Szeged) 50 (1986), 337-350.

[2] Z. Daróczy, I. Kátai, On functions additive with respect to interval flling sequences. Acta Math. Hungar. 51 (1988), no. 1-2, 185-200.

[3] P. Erdôs, I. Joó, V. Komornik, Characterization of the unique expansions $1=$ $\sum q^{-n_{i}}$ and related problems, Bull. Soc. Math. France 118 (1990), 377-390.

[4] K.-H. Indlekofer, I. Kátai and P. Racskó, Number systems and fractal geometry, Probability theory and applications, Essays to the Mem. of J. Mogyoródi, Math. Appl. 80 (1992), 319-334.

[5] S. Kakeya, On the set of partial sums of an infinite series, Proc. Tokyo Math.Phys. Soc (2) 7 (1914), 250-251.

[6] S. Kakeya, On the partial sums of an infinite series, Tôhoku Sc. Rep. 3 (1915), 159-163.

[7] D. M. Kane, Generalized base representations, J. Number Theory 120 (2006), 92-100.

[8] A. Pethó, Notes on CNS polynomials and integral interpolation, In: More Sets, Graphs and Numbers, Eds.: E. Gyốry, G. O. H. Katona and L. Lovász, Bolyai Soc. Math. Stud., 15, Springer, Berlin, 2006, 301-315.

[9] G. Pólya, G. Szegô, Problems and Exercises in Analysis, Vol. I, SpringerVerlag, Berlin, New York, 1972.

[10] A. Rényi, Representations for real numbers and their ergodic properties, Acta Math. Hungar. 8 (1957), 477-493.

Département de mathématique, Université de Strasbourg, 7 Rue René Descartes, 67084 Strasbourg Cedex, France

E-mail address: vilmos.komornik@math.unistra.fr

University of Debrecen, Department of Computer Science, University of Debrecen, H-4010 Debrecen P.O. Box 12, Hungary

E-mail address: petho.attila@inf.unideb.hu 